\title{
Thermal Creep and the Skyrmion Hall Angle in Driven Skyrmion Crystals
}

\author{
C. Reichhardt and C. J. O. Reichhardt \\ Theoretical Division and Center for Nonlinear Studies, \\ Los Alamos National Laboratory, \\ Los Alamos, New Mexico 87545, USA
}

(Dated: May 24, 2018)

\begin{abstract}
We numerically examine thermal effects on the skyrmion Hall angle for driven skyrmions interacting with quenched disorder. We identify a creep regime in which motion occurs via intermittent jumps between pinned and flowing states. Here the skyrmion Hall angle is zero since the skyrmions have time to relax into equilibrium positions in the pinning sites, eliminating the side-jump motion induced by the Magnus force. At higher drives we find a crossover to a viscous flow regime where the skyrmion Hall angle is finite and increases with increasing drive or temperature. Our results are in agreement with recent experiments which also show a regime of finite skyrmion velocity with zero skyrmion Hall angle crossing over to a viscous flow regime with a skyrmion Hall angle that increases with drive.
\end{abstract}

A wide range of systems with quenched disorder exhibit depinning phenomena under an external drive [1, 2], including sliding charge density waves [3], vortices in type-II superconductors [4], colloids [5, 6] and magnetic domain walls [7]. At zero temperature, these systems have a well defined depinning threshold $F_{c}$ below which motion does not occur, while at finite temperature, a finite velocity arises at much lower drives due to thermal creep effects [1]. Another system that exhibits depinning phenomena is skyrmions in chiral magnets [8-10]. Skyrmions are topological particle-like magnetic objects that can form lattices and be driven by an applied current or by other methods [8, 11, 12]. Skyrmion velocityforce relations can be obtained by transport measures [9, 13] and direct imaging [14 17]. The topology of the skyrmions makes them unique among systems that exhibit depinning since the skyrmion dynamics is strongly influenced by the Magnus force, which generates a velocity component perpendicular to the forces experienced by the skyrmion [8].

One consequence of the Magnus force is that skyrmions move at an angle, called the skyrmion Hall angle $\theta_{s k}$, with respect to an external driving force [8, 16 18]. The intrinsic value of $\theta_{s k}$ is determined by the ratio of the Magnus term to the damping term, and in the absence of quenched disorder, $\theta_{s k}$ is a constant. Particle-based simulations of moving skyrmions interacting with quenched disorder have shown that $\theta_{s k}$ can vary, starting at $\theta_{s k}=0$ at depinning, increasing with increasing velocity, and saturating to the defect-free intrinsic value at higher drives [19 22]. This effect arises due to the Magnusinduced side jump experienced by the skyrmions when they move through a pinning site [20, 23]. Under rapid skyrmion motion, the magnitude of the side jumps is reduced since the skyrmion does not have time to respond fully to the pinning potential before exiting the pinning site. Continuum-based simulations reveal a similar drive dependence of $\theta_{s k}$ when disorder is present, with $\theta_{s k}$ remaining constant in the absence of disorder [24, 25]. In imaging experiments of skyrmions under an external drive, $\theta_{s k}=0$ at low drives just above depinning, but as the drive increases, $\theta_{s k}$ increases linearly until it reaches a saturation at high drives close to the predicted intrinsic value [16]. In other experiments which show a similar drive dependence of $\theta_{s k}$, it was argued that in addition to the pinning interactions, excitation of internal modes of the skyrmions or a change in skyrmion size with driving can modify the skyrmion Hall angle [17]. In more recent work it was claimed that both of these effects contribute to changes in $\theta_{s k}$ 26]. Far less is known about how thermal creep affects skyrmion motion or the value of $\theta_{s k}$. Thermal effects are relevant since many materials support skyrmions at room temperature [14 16, 18, 27, 28]. Additionally, in recent experiments, thermally induced Brownian motion of skyrmions has been observed directly in the absence of an external drive [29].

In this work we examine the thermal creep of skyrmions and its effect on $\theta_{s k}$ using a particle-based skyrmion model. Thermal motion is most significant near the depinning threshold where the drive is small, in a regime where the effect of the skyrmion shape on $\theta_{s k}$, which has been proposed to be important at high drives [17], is minimal. We find that the depinning threshold decreases with increasing temperature and that a creep regime appears which is characterized by skyrmions jumping or even avalanching between pinned and moving states. Within the creep regime, $\theta_{s k}=0$, while at higher drives there is a crossover to viscous flow where the skyrmions are always moving and the value of $\theta_{s k}$ becomes finite and increases with drive. We note that recent particle-based simulations have examined thermal effects in skyrmions, but these studies focused on the aging dynamics in the absence of a drive [30].

Simulation- We consider a two-dimensional system of rigid skyrmions with periodic boundary conditions in the $x$ - and $y$-directions. The skyrmion dynamics are mod- 


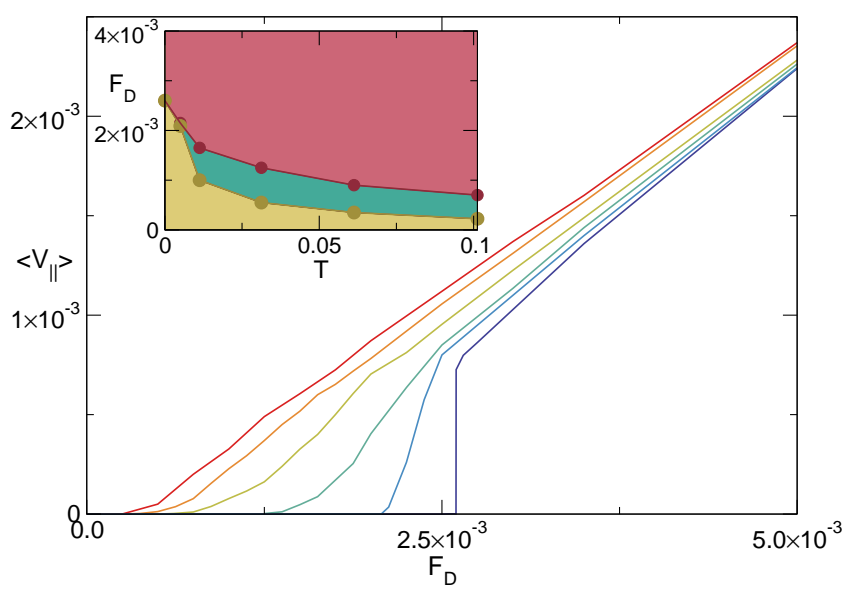

FIG. 1: The time average skyrmion velocity in the direction parallel to the drive, $\left\langle V_{\|}\right\rangle$, vs $F_{D}$ at temperatures $T=0.0$ (dark blue), 0.005 (light blue), 0.01125 (dark green), 0.03125 (light green), 0.06125 (orange), and 0.1025 (red). As $T$ increases, there is a decrease in the depinning threshold $F_{c}$ and the behavior of $\left\langle V_{\| l}\right\rangle$ becomes increasingly nonlinear. Inset: Dynamic phase diagram as a function of $F_{D}$ vs $T$ highlighting the pinned (yellow), creep (green), and viscous flow (pink) regimes.

eled using a modified version of the Thiele equation 19 21, 31], and we include Langevin dynamics that are similar to those employed previously to model thermal relaxation effects in skyrmions [30]. The equation of motion of skyrmion $i$ is $\alpha_{d} \mathbf{v}_{i}+\alpha_{m} \hat{z} \times \mathbf{v}_{i}=\mathbf{F}_{i}^{s s}+\mathbf{F}_{i}^{p}+\mathbf{F}^{D}+\mathbf{F}_{i}^{T}$, where $\mathbf{v}_{i}=d \mathbf{r}_{i} / d t$ is the skyrmion velocity and $\alpha_{d}$ and $\alpha_{m}$ are the damping and Magnus terms, respectively. The intrinsic skyrmion Hall angle is given by $\theta_{s k}^{i n}=\tan ^{-1}\left(\alpha_{m} / \alpha_{d}\right)$. We fix $\alpha_{m} / \alpha_{d}=1.0$ so that $\theta_{s k}^{i n}=45^{\circ}$, which is close to the value in recent experiments [16, 17]. The skyrmion-skyrmion interaction force $\mathbf{F}_{i}^{s s}=\sum_{j \neq i}^{N} K_{1}\left(r_{i j}\right) \hat{\mathbf{r}}_{i j}$, where $K_{1}$ is the modified Bessel function, $r_{i j}=\left|\mathbf{r}_{i}-\mathbf{r}_{j}\right|$ is the distance between skyrmions $i$ and $j, \hat{\mathbf{r}}_{i j}=\left(\mathbf{r}_{i}-\mathbf{r}_{j}\right) / r_{i j}$, and $N$ is the number of skyrmions in the sample [19, 31, 32]. The pinning force $\mathbf{F}_{i}^{s p}=\sum_{j=1}^{N_{p}}\left(F_{p} / r_{p}\right)\left(\mathbf{r}_{i}-\mathbf{r}_{p}^{j}\right) \Theta\left(r_{p}-\left|\mathbf{r}_{i}-\mathbf{r}_{p}^{j}\right|\right)$ arises from randomly placed non-overlapping parabolic traps of radius $r_{p}=0.15$ at locations $\mathbf{r}_{p}^{j}$ that can exert a maximum pinning force of $F_{p}=0.03$ on a skyrmion. Here $\Theta$ is the Heaviside step function. The driving force from an applied current is $\mathbf{F}^{D}=F_{D} \hat{\mathbf{x}}$, and we measure the average velocity per skyrmion both parallel, $\left\langle V_{\|}\right\rangle=N^{-1} \sum_{i=1}^{N} \mathbf{v}_{i} \cdot \hat{\mathbf{x}}$, and perpendicular, $\left\langle V_{\perp}\right\rangle=N^{-1} \sum_{i=1}^{N} \mathbf{v}_{i} \cdot \hat{\mathbf{y}}$, to the drive. The skyrmion Hall angle is given by $\theta_{s k}=\tan ^{-1}\left(\left\langle V_{\perp}\right\rangle /\left\langle V_{||}\right\rangle\right)$. The stochastic thermal force $\mathbf{F}_{i}^{T}$ has the properties $\left\langle\mathbf{F}_{i}^{T}\right\rangle=0$ and $\left\langle\mathbf{F}_{i}^{T}(t) \mathbf{F}_{j}^{T}\left(t^{\prime}\right)\right\rangle=2 \eta k_{B} T \delta_{i j} \delta\left(t-t^{\prime}\right)$. Here we set $\eta=1.0$ and $k_{B}=1.0$. We use a skyrmion density of $n_{s}=N / L^{2}=0.16$, where the sample is of size $L \times L$ with $L=36$. We fix the pinning density at $n_{p}=N_{p} / L^{2}=0.2$.

Results - In Fig. 1 we plot $\left\langle V_{\|}\right\rangle$versus $F_{D}$ for tempera-

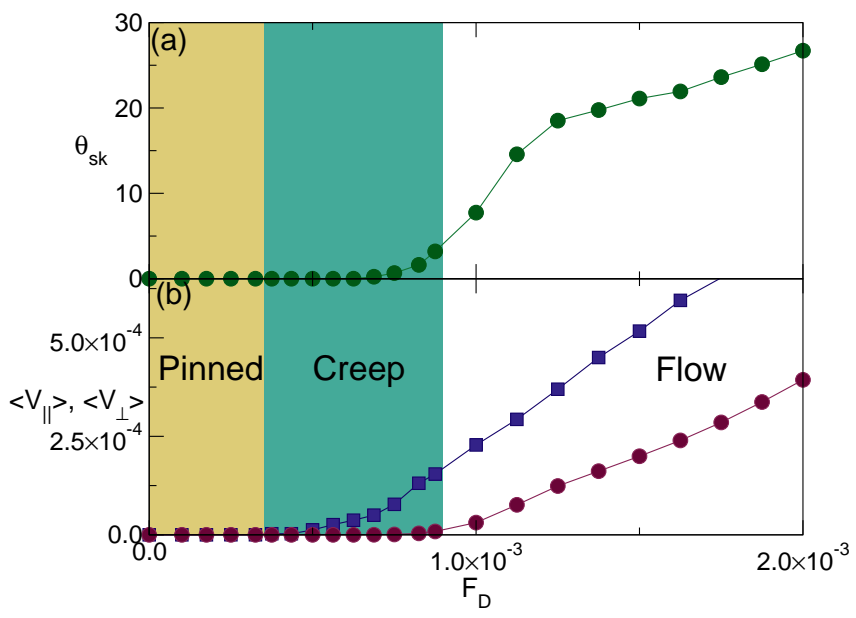

FIG. 2: (a) $\theta_{s k}$ vs $F_{D}$ at $T=0.06125$. (b) $\left\langle V_{\|}\right\rangle$(blue squares) and $\left\langle V_{\perp}\right\rangle$ (red circles) vs $F_{D}$ in the same system. Here there is a pinned phase, a creep phase in which $\theta_{s k}$ is close to zero, and a flowing phase.

tures ranging from $T=0$ to $T=0.1025$. At $T=0$, there is a sharp depinning threshold at a critical depinning force of $F_{c}=2.6 \times 10^{-3}$. As $T$ increases, $F_{c}$ decreases and the regime of nonlinear behavior of $\left\langle V_{\|}\right\rangle$grows in extent. We measure the evolution of $\theta_{s k}$ at each temperature as a function of drive. Previous work at $T=0$ showed that $\theta_{s k}$ is zero only in the pinned phase, and that $\theta_{s k}$ increases with increasing $F_{D}$ for $F_{D} / F_{c}>1.0$ 20 22]. At finite temperature we find an extended creep regime in which $\left\langle V_{\|}\right\rangle>0$ while $\left\langle V_{\perp}\right\rangle=0$, giving $\theta_{s k}=0$ even though the skyrmion velocity is finite. Within the creep regime, the skyrmion motion is intermittent and consists of jumps between moving and pinned states. At higher drives, the creep regime transitions into a viscous flow phase in which the skyrmions are always in motion and $\theta_{s k}$ increases with $F_{D}$. Based on measurements of $\left\langle V_{\|}\right\rangle,\left\langle V_{\perp}\right\rangle, \theta_{s k}$, and histograms of the skyrmion velocities, we construct a dynamic phase diagram as a function of $F_{D}$ versus $T$ highlighting the pinned phase with $\left\langle V_{\|}\right\rangle=\left\langle V_{\perp}\right\rangle=0$, the creep phase with $\left\langle V_{\|}\right\rangle>0$ and $\left\langle V_{\perp}\right\rangle=0$, and the viscous flow regime with $\left\langle V_{\|}\right\rangle>0$ and $\left\langle V_{\perp}\right\rangle>0$, as shown in the inset of Fig. 1

In Fig. 2(a) we plot $\theta_{s k}$ versus $F_{D}$ at $T=0.06125$, and in Fig. 2(b) we show the corresponding $\left\langle V_{\|}\right\rangle$and $\left\langle V_{\perp}\right\rangle$ versus $F_{D}$ curves. In the pinned phase, both $\left\langle V_{\|}\right\rangle$and $\left\langle V_{\perp}\right\rangle$ are zero, while in the creep regime, $\left\langle V_{\| \mid}\right\rangle$is finite but $\left\langle V_{\perp}\right\rangle$ is zero or nearly zero, so that $\theta_{s k}$ is zero or close to zero. In the flow regime, both velocity components increase and $\theta_{s k}$ rises from zero. When the system first enters the flow regime, the increase in $\theta_{s k}$ with $F_{D}$ is nonlinear, but there is a crossover to a linear increase in $\theta_{s k}$ at higher drives. Above the range of drives illustrated in Fig. 2, $\theta_{s k}$ saturates to the intrinsic value of $\theta_{s k}^{i n}=45^{\circ}$. The pinned, creep, and flow behavior of $\left\langle V_{\|}\right\rangle,\left\langle V_{\perp}\right\rangle$, and $\theta_{s k}$ is very similar to the observations in imaging experi- 


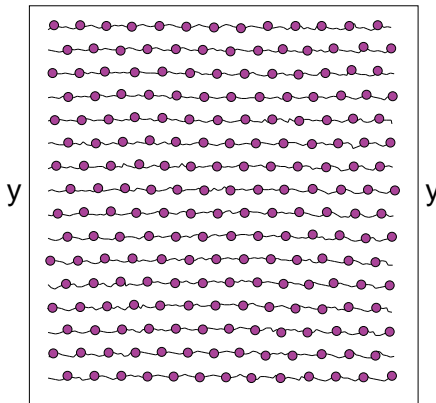

(a)

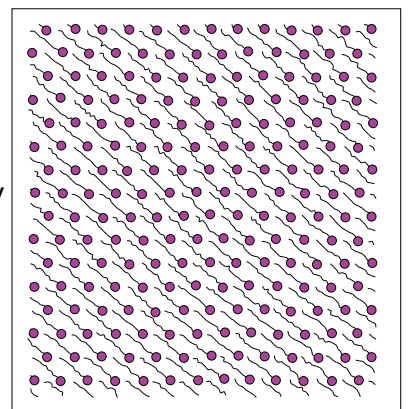

(b)
FIG. 3: The skyrmion positions (dots) and trajectories (lines) for the system in Fig. 2 at $T=0.06125$. (a) The creep phase at $F_{D}=7.5 \times 10^{-4}$, where $\theta_{s k}=0$ and the skyrmions move in the direction of drive. (b) The flow phase at $F_{D}=3.5 \times 10^{-3}$, where $\theta_{s k}=35^{\circ}$.
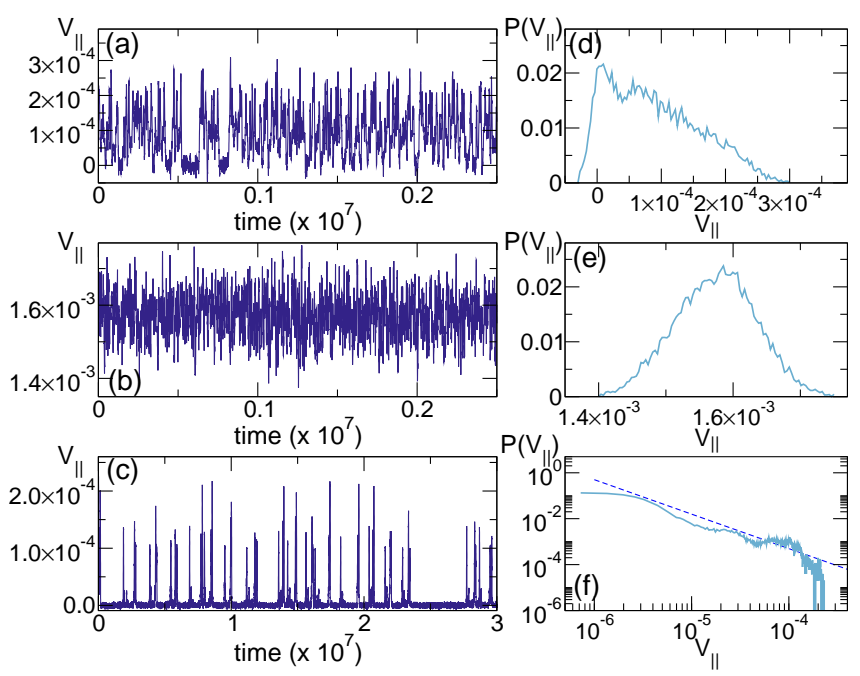

FIG. 4: (a) Time series of $V_{\|}(t)$ in units of $10^{7}$ simulation time steps in the creep regime at $F_{D}=7.5 \times 10^{-4}$ and $T=0.06125$. (b) The corresponding velocity histogram $P\left(V_{\|}\right)$. (c) $V_{\|}(t)$ and (d) $P\left(V_{\|}\right)$in the flow regime at $F_{D}=3.5 \times 10^{-3}$ and $T=0.06125$, where $\left\langle V_{\|}\right\rangle>0$ and $\left\langle V_{\perp}\right\rangle>0$. (e) $V_{\|}(t)$ in the creep regime at $F_{D}=1.5 \times 10^{-3}$ and $T=0.01125$, where motion occurs in the form of avalanches. (f) The corresponding histogram $P\left(V_{\|}\right)$, where the dashed line is a power law fit to $P\left(V_{\|}\right) \propto V_{\|}^{-\alpha}$ with $\alpha=1.5$.

ments of the room temperature motion of skyrmions [16]. In Fig. 3(a) we show the skyrmion positions and trajectories for the system in Fig. 2 in the creep regime at $T=0.06125$ and $F_{D}=7.5 \times 10^{-4}$, where the skyrmions move in the direction of drive. At the same temperature but at a higher drive of $F_{D}=3.5 \times 10^{-3}$ in Fig. [3), the skyrmions are in the flow regime and move at an angle of $\theta_{s k}=35^{\circ}$ with respect to the drive.

The skyrmion motion within the creep phase has a stop-start character, where a skyrmion that is trapped by a pin escapes from the pin and undergoes a brief interval of motion before becoming trapped by an adjacent pin. While trapped, the skyrmion spirals toward the equilibrium location in the pinning site at which the pinning and driving forces are exactly balanced. In Fig. 4(a,b) we plot a velocity time series $V_{\|}(t)$ and corresponding velocity histogram $P\left(V_{\|}\right)$in the creep regime at $F_{D}=7.5 \times 10^{-4}$ and $T=0.06125$. There is a peak in $P\left(V_{\|}\right)$at $V_{\|}=0$, indicating that the skyrmions spend the largest portion of their time trapped in pinning sites. In the flow regime, shown in Fig. 4(c,d) at $F_{D}=3.5 \times 10^{-3}$ and $T=0.06125$, the skyrmions are constantly flowing and $V_{\|}>0$ at all times.

In the creep regime, $\theta_{s k}=0^{\circ}$ because the Magnus force is a dynamical quantity that can act only when the skyrmions are driven out of equilibrium. If there is sufficient time between jumps of the skyrmion from one pinning site to another, the skyrmion gradually spirals to a position in the pinning site where the pinning and drive forces are balanced. The location of this point is independent of the Magnus term, so over long times, a skyrmion in the strictly overdamped limit of $\alpha_{m}=0$ settles into the same position as a skyrmion with $\alpha_{m}>0$. On the other hand, if the skyrmions are continuously moving over the pinning sites, they experience a side jump motion generated by the Magnus force that pushes them away from the equilibrium force balance point of the pin, producing a finite value of $\theta_{s k}$ with a magnitude that depends on the strength of the Magnus force. As $F_{D}$ increases and the skyrmions move faster, the perturbation of the Magnus force by the pinning is reduced and $\theta_{s k}$ increases until it reaches the intrinsic value $\theta_{s k}=\theta_{s k}^{i n}$. For increasing temperature $T$ at fixed $F_{D}$, thermal fluctuations smear out the effectiveness of the pinning, permitting the skyrmions to move continuously and causing $\theta_{s k}$ to increase.

If the temperature is lowered, within the creep regime the skyrmions spend an even larger fraction of their time trapped in pinning sites, the motion becomes increasingly intermittent, and avalanches dominate the behavior, as illustrated in the plot of $V_{\|}(t)$ in Fig. 4(e) at $F_{D}=1.5 \times 10^{-3}$ and $T=0.01125$. In Fig. 4(f) we show $P\left(V_{\|}\right)$in the avalanche regime on a log-log scale, where the dashed line is a power law fit to $P\left(V_{\|}\right) \propto V_{\|}^{-\alpha}$ with $\alpha=1.5$. In previous work, we examined the avalanche behavior just at the depinning threshold for a $T=0$ system, and found that various quantities such as the avalanche size and duration are power law distributed with exponents similar to that in Fig. 4(f) 32]. Here we find that under finite temperature in the creep regime, the thermal fluctuations lower $F_{c}$ but the system can still exhibit critical behavior near the depinning threshold. These results indicate that in certain cases, thermally induced skyrmion avalanches can be realized with a fixed current.

In Fig. 5 we illustrate the temperature dependence of $\theta_{s k}$ at a fixed $F_{D}$ in two regimes: $F_{D}>F_{c}$ where the 


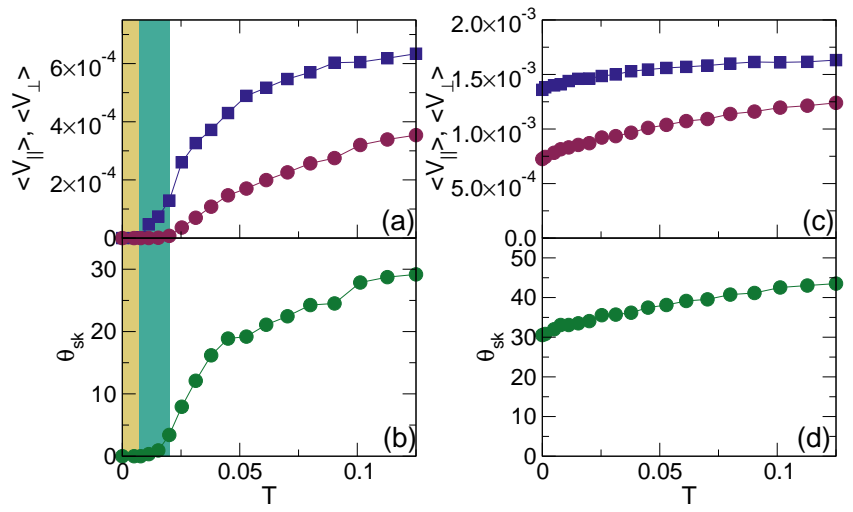

FIG. 5: (a) $\left\langle V_{\|}\right\rangle$(blue squares) and $\left\langle V_{\perp}\right\rangle$ (red circles) vs $T$ at $F_{D}=1.5 \times 10^{-3}$. (b) The corresponding $\theta_{s k}$ vs $T$. Yellow shading indicates the pinned regime, green shading is the creep state, and the unshaded area is in the flowing state. (c) $\left\langle V_{\|}\right\rangle$(blue squares) and $\left\langle V_{\perp}\right\rangle$ (red circles) vs $T$ for the same system in the flowing state at $F_{D}=3.5 \times 10^{-3}$. (d) The corresponding $\theta_{s k}$ vs $T$ curve shows a linear increase in $\theta_{s k}$ with increasing $T$ up to the intrinsic value of $\theta_{s k}^{i n}=45^{\circ}$.

skyrmions are always moving, and $F_{D}<F_{c}$ where the skyrmions spend most of their time pinned. In Fig. [5(a) we plot $\left\langle V_{\|}\right\rangle$and $\left\langle V_{\perp}\right\rangle$ versus $T$ at $F_{D}=1.5 \times 10^{-3}$, a drive that is lower than the $T=0$ depinning thresh-

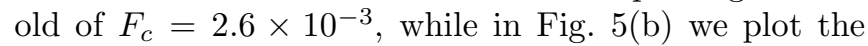
corresponding $\theta_{s k}$ versus $T$. We find a pinned state for $T>0.00725$ and a creep state for $0.00725 \leq T<0.02$. For $T \geq 0.02$, the system is in a flowing phase and $\theta_{s k}$ increases with increasing $T$. In Fig. 5 (c) we show $\left\langle V_{\|}\right\rangle$ and $\left\langle V_{\perp}\right\rangle$ versus $T$ at $F_{D}=3.5 \times 10^{-3}$, a drive that is higher than the $T=0$ value of $F_{c}$, and in Fig. 5 (d) we plot the corresponding $\theta_{s k}$ versus $T$. At this drive, the skyrmions are in the flowing state for all values of $T$. The skyrmion Hall angle $\theta_{s k}=31^{\circ}$ at $T=0$ and increases roughly linearly with increasing temperature before saturating at the intrinsic value of $\theta_{s k}^{i n}=45^{\circ}$ at high temperatures. This indicates that a temperature dependence of $\theta_{s k}$ persists even in the viscous flow phase. We note that in the absence of pinning, $\theta_{s k}$ is independent of both $F_{D}$ and $T$ within the particle model. In continuum simulations performed without pinning, $\theta_{s k}$ was also shown to be independent of the drive amplitude [24].

Summary - We have examined the effect of temperature on skyrmion creep and motion using a particle based model. As temperature increases, we find that the depinning threshold drops and a nonlinear creep regime appears at low drives. Within the creep regime, the skyrmion Hall angle is zero and the skyrmions spend most of their time trapped in pinning sites, making occasional hops from one pinning site to another. While trapped, the skyrmions have enough time to spiral to the equilibrium position in the pin at which the drive and pinning forces are balanced, eliminating the Magnus- induced side-jump motion that would give a finite value for the skyrmion Hall angle. In the flowing regime, the skyrmions are always moving and the skyrmion Hall angle increases linearly with increasing drive. At low temperatures in the creep regime, we find that skyrmion motion occurs in the form of avalanches, and that the skyrmion velocities are power law distributed. The effect of temperature on the skyrmion Hall angle is most pronounced at small drives where thermally-induced depinning occurs; however, even within the flowing phase, the skyrmion Hall angle increases with increasing temperature.

We gratefully acknowledge the support of the U.S. Department of Energy through the LANL/LDRD program for this work. This work was carried out under the auspices of the NNSA of the U.S. DoE at LANL under Contract No. DE-AC52-06NA25396.

[1] D. S. Fisher, Collective transport in random media: From superconductors to earthquakes, Phys. Rep. 301, 113 (1998).

[2] C. Reichhardt and C. J. Olson Reichhardt, Depinning and nonequilibrium dynamic phases of particle assemblies driven over random and ordered substrates: A review, Rep. Prog. Phys. 80, 026501 (2017).

[3] G. Grüner, The dynamics of charge-density waves, Rev. Mod. Phys. 60, 1129 (1988).

[4] G. Blatter, M. V. Feigel'man, V. B. Geshkenbein, A. I. Larkin, and V. M. Vinokur, Vortices in high-temperature superconductors, Rev. Mod. Phys. 66, 1125 (1994).

[5] C. Reichhardt and C. J. Olson, Colloidal dynamics on disordered substrates, Phys. Rev. Lett. 89, 078301 (2002).

[6] T. Bohlein, J. Mikhael, and C. Bechinger, Observation of kinks and antikinks in colloidal monolayers driven across ordered surfaces, Nat. Mater. 11, 126 (2012).

[7] P. J. Metaxas, J. P. Jamet, A. Mougin, M. Cormier, J. Ferré, V. Baltz, B. Rodmacq, B. Dieny, and R. L. Stamps, Creep and flow regimes of magnetic domain-wall motion in ultrathin $\mathrm{Pt} / \mathrm{Co} / \mathrm{Pt}$ Films with perpendicular anisotropy, Phys. Rev. Lett. 99, 217208 (2007).

[8] N. Nagaosa and Y. Tokura, Topological properties and dynamics of magnetic skyrmions, Nat. Nanotechnol. 8, 899 (2013).

[9] T. Schulz, R. Ritz, A. Bauer, M. Halder, M. Wagner, C. Franz, C. Pfleiderer, K. Everschor, M. Garst, and A. Rosch, Emergent electrodynamics of skyrmions in a chiral magnet, Nat. Phys. 8, 301 (2012).

[10] J. Iwasaki, M. Mochizuki, and N. Nagaosa, Universal current-velocity relation of skyrmion motion in chiral magnets, Nat. Commun. 4, 1463 (2013).

[11] S. Mühlbauer, B. Binz, F. Jonietz, C. Pfleiderer, A. Rosch, A. Neubauer, R. Georgii, and P. Böni, Skyrmion lattice in a chiral magnet, Science 323, 915 (2009).

[12] X. Z. Yu, Y. Onose, N. Kanazawa, J. H. Park, J. H. Han, Y. Matsui, N. Nagaosa, and Y. Tokura, Real-space observation of a two-dimensional skyrmion crystal, Nature (London) 465, 901 (2010). 
[13] D. Liang, J.P. DeGrave, M.J. Stolt, Y. Tokura, and S. Jin, Current-driven dynamics of skyrmions stabilized in MnSi nanowires revealed by topological Hall effect, Nat. Commun. 6, 8217 (2015).

[14] W. Jiang, P. Upadhyaya, W. Zhang, G. Yu, M. B. Jungfleisch, F. Y. Fradin, J. E. Pearson, Y. Tserkovnyak, K. L. Wang, O. Heinonen, S. G. E. te Velthuis, and A. Hoffmann, Blowing magnetic skyrmion bubbles, Science 349, 283 (2015).

[15] S. Woo, K. Litzius, B. Krüger, M.-Y. Im, L. Caretta, K. Richter, M. Mann, A. Krone, R. M. Reeve, M. Weigand, P. Agrawal, I. Lemesh, M.-A. Mawass, P. Fischer, M. Kläui, and G. S. D. Beach, Observation of room-temperature magnetic skyrmions and their currentdriven dynamics in ultrathin metallic ferromagnets, Nat. Mater. 15, 501 (2016).

[16] W. Jiang, X. Zhang, G. Yu, W. Zhang, X. Wang, M. B. Jungfleisch, J. E. Pearson, X. Cheng, O. Heinonen, K. L. Wang, Y. Zhou, A. Hoffmann, and S. G. E. te Velthuis, Direct observation of the skyrmion Hall effect, Nat. Phys. 13, 162 (2017).

[17] K. Litzius, I. Lemesh, B. Krüger, P. Bassirian, L. Caretta, K. Richter, F. Büttner, K. Sato, O.A. Tretiakov, J. Förster, R.M. Reeve, M. Weigand, I. Bykova, H. Stoll, G. Schütz, G.S.D. Beach, and M. Kläui, Skyrmion Hall effect revealed by direct time-resolved X-ray microscopy, Nat. Phys. 13, 170 (2017).

[18] R. Tolley, S. A. Montoya, and E. E. Fullerton, Room-temperature observation and current control of skyrmions in $\mathrm{Pt} / \mathrm{Co} / \mathrm{Os} / \mathrm{Pt}$ thin films, Phys. Rev. Materials 2, 044404 (2018).

[19] C. Reichhardt, D. Ray, and C. J. Olson Reichhardt, Collective transport properties of driven skyrmions with random disorder, Phys. Rev. Lett. 114, 217202 (2015).

[20] C. Reichhardt, D. Ray, and C. J. Olson Reichhardt, Quantized transport for a skyrmion moving on a twodimensional periodic substrate, Phys. Rev. B 91, 104426 (2015).

[21] C. Reichhardt and C.J.O. Reichhardt, Noise fluctuations and drive dependence of the skyrmion Hall effect in disordered systems, New J. Phys. 18, 095005 (2016).

[22] S.A. Díaz, C.J.O. Reichhardt, D.P. Arovas, A. Saxena, and C. Reichhardt, Fluctuations and noise signatures of driven magnetic skyrmions, Phys. Rev. B 96, 085106 (2017).

[23] J. Müller and A. Rosch, Capturing of a magnetic skyrmion with a hole, Phys. Rev. B 91, 054410 (2015).

[24] W. Legrand, D. Maccariello, N. Reyren, K. Garcia, C. Moutafis, C. Moreau-Luchaire, S. Collin, K. Bouzehouane, V. Cros, and A. Fert, Room-temperature current-induced generation and motion of sub-100 nm skyrmions, Nano Lett. 17, 2703 (2017).

[25] J.-V. Kim and M.-W. Yoo, Current-driven skyrmion dynamics in disordered films, Appl. Phys. Lett. 110, 132404 (2017).

[26] K. Litzius, P. Bassirian, J. Leliaert, S. Kromin, J. Zazvorka, I. Lemesh, N. Kerber, A. Churikova, D. Heinze, N. Keil, M. Weigand, G. Schütz, G. S. D. Beach, and M. Kläui, to be published.

[27] Y. Tokunaga, X.Z. Yu, J.S. White, H.M. Rønnow, D. Morikawa, Y. Taguchi, and Y. Tokura, A new class of chiral materials hosting magnetic skyrmions beyond room temperature, Nat. Commun. 6, 7638 (2015).

[28] A. Soumyanarayanan, M. Raju, A.L. Gonzalez-Oyarce, A.K.C. Tan, M.-Y. Im, A.P. Petrović, P. Ho, K.H. Khoo, M. Tran, C.K. Gan, F. Ernult, and C. Panagopoulos, Tunable room-temperature magnetic skyrmions in $\mathrm{Ir} / \mathrm{Fe} / \mathrm{Co} / \mathrm{Pt}$ multilayers, Nat. Mater. 16, 898 (2017).

[29] J. Zázvorka, F. Jakobs, D. Heinze, N. Keil, S. Kromin, S. Jaiswal, K. Litzius, G. Jakob, P. Virnau, D. Pinna, K. Everschor-Sitte, A. Donges, U. Nowak, and M. Kläui, Thermal skyrmion diffusion applied in probabilistic computing, arXiv:1805.05924

[30] B. L. Brown, U. C. Täuber, and M. Pleimling, Effect of the Magnus force on skyrmion relaxation dynamics, Phys. Rev. B 97, 020405(R) (2018).

[31] S.-Z. Lin, C. Reichhardt, C.D. Batista, and A. Saxena, Particle model for skyrmions in metallic chiral magnets: Dynamics, pinning, and creep, Phys. Rev. B 87, 214419 (2013).

[32] S.A. Díaz, C. Reichhardt, D.P. Arovas, A. Saxena, and C.J.O. Reichhardt, Avalanches and criticality in driven magnetic skyrmions, Phys. Rev. Lett. 120, 117203 (2018). 